\title{
Peak Point Theorems for Uniform Algebras on Smooth Manifolds
}

\author{
John T. Anderson and Alexander J. Izzo
}

\begin{abstract}
It was once conjectured that if $A$ is a uniform algebra on its maximal ideal space $X$, and if each point of $X$ is a peak point for $A$, then $A=C(X)$. This peak point conjecture was disproved by Brian Cole in 1968. However, Anderson and Izzo showed that the peak point conjecture does hold for uniform algebras generated by smooth functions on smooth two-manifolds with boundary. The corresponding assertion for smooth three-manifolds is false, but Anderson, Izzo, and Wermer established a peak point theorem for polynomial approximation on realanalytic three-manifolds with boundary. Here we establish a more general peak point theorem for real-analytic three-manifolds with boundary analogous to the two-dimensional result. We also show that if $A$ is a counterexample to the peak point conjecture generated by smooth functions on a manifold of arbitrary dimension, then the essential set for $A$ has empty interior.
\end{abstract}

\section{Introduction}

Let $A$ be a uniform algebra on a compact metric space $X$. That is, $A$ is a subalgebra of $C(X)$ (the algebra of all complex-valued continuous functions on $X$ ) that is closed in the supremum norm, contains the constants, and separates the points of $X$. A central problem in the subject of uniform algebras is to characterize $C(X)$ among the uniform algebras on $X$. One necessary condition for $A$ to be $C(X)$ is that the maximal ideal space of $A$ be $X$, that is, the only nonzero multiplicative linear functionals on $A$ be the point evaluations. Another necessary condition is that every point of $X$ be a peak point for $A$. (A point $p \in X$ is a peak point for $A$ if there exists a function $f \in A$ with $f(p)=1$ while $|f|<1$ on $X \backslash\{p\}$.) It was once conjectured that together these two necessary conditions for $A=C(X)$ were also sufficient. However, a counterexample to this "peak point conjecture" was produced by Brian Cole in his 1968 thesis (see [4, Appendix] or [12, Section 19]). Subsequently

2000 Mathematics Subject Classification. 46J10, 46J15. 
other counterexamples were given; the one that is probably the simplest is due to Richard Basener [3].

In 2001 Anderson and Izzo [1] established a peak point theorem for uniform algebras on two-dimensional manifolds: if $A$ is a uniform algebra generated by $C^{1}$ functions on a compact $C^{1}$ two-dimensional manifold with boundary $M$ such that the maximal ideal space of $A$ is $M$ and each point of $M$ is a peak point for $A$, then $A=C(M)$. The corresponding assertion for three-dimensional manifolds is false: there is a counterexample to the peak point conjecture due to Izzo [8] generated by $C^{\infty}$ functions on a smooth solid torus, and a counterexample generated by $C^{\infty}$ functions on the 3 -sphere can easily be obtained from Basener's example (see [1]). Nevertheless Anderson, Izzo, and Wermer [2] proved a peak point theorem for polynomial approximation on real-analytic three-dimensional submanifolds of $\mathbf{C}^{n}$. In the present paper we generalize this result to general uniform algebras generated by real-analytic functions on real-analytic three-dimensional manifolds. The precise result is as follows.

Theorem 1.1: Let $\Sigma$ be a real-analytic three-dimensional manifold with boundary, and let $X$ be a compact subset of $\Sigma$ such that $\partial X$ (the boundary of $X$ relative to $\Sigma$ ) is a twodimensional submanifold of $\Sigma$ of class $C^{1}$. Let $A$ be a uniform algebra on $X$ generated by a collection $\Phi$ of functions that are real-analytic on a neighborhood of $X$. If the maximal ideal space of $A$ is $X$ and every point of $X$ is a peak point for $A$, then $A=C(X)$.

Perhaps the notion of the boundary $\partial X$ of $X$ relative to $\Sigma$ should be made more explicit; $\partial X$ denotes the union of the topological boundary of $X$ relative to $\Sigma$ and the set $X \cap \partial \Sigma$.

We will also prove a peak point theorem of a different flavor from all of the results discussed above by showing that a uniform algebra generated by $C^{1}$ functions on a manifold $M$ and satisfying the hypotheses of the peak point conjecture must in a certain sense be almost $C(M)$. The essential set $E$ for a uniform algebra $A$ on a space $X$ is the smallest 
closed subset of $X$ such that $A$ contains every continuous function on $X$ that vanishes on $E$. Note that $A$ contains every continuous function whose restriction to $E$ lies in the restriction $A \mid E$ of $A$ to $E$. The uniform algebra $A$ is said to be essential if $E=X$.

Theorem 1.2: Let $A$ be a uniform algebra on a compact $C^{1}$ manifold with boundary $M$. Assume that $A$ is generated by $C^{1}$ functions, that the maximal ideal space of $A$ is $M$, and that every point of $M$ is a peak point for $A$. Then the uniform algebra $A$ is not essential. In fact, the essential set for $A$ has empty interior in $M$.

This result is particularly interesting because it requires only $C^{1}$ smoothness and applies in all dimensions. It shows that for uniform algebras generated by $C^{1}$ functions on a manifold with boundary, the hypotheses of the peak point conjecture imply that the functions in the algebra are arbitrary except on a small set. The result has already been used by Izzo [9] to answer a question about uniform algebras on spheres raised by Ronald Douglas in connection with a conjecture of William Arveson.

The proof of Theorem 1.1 parallels closely the proof of the special case in [2]. That proof relied on a theorem of Hörmander and Wermer [7] (which under a weaker smoothness hypothesis is due to O'Farrell, Preskenis, and Walsh [11]) regarding approximation and complex tangents. In the proof of Theorem 1.1, that result will be replaced by a recent generalization due to Izzo [10, Theorem 1.3]. This result will also be needed for the proof of Theorem 1.2. The needed result is the following.

Theorem 1.3: Let $A$ be a uniform algebra on a compact Hausdorff space $X$. Suppose that the maximal ideal space of $A$ is $X$. Suppose also that $E$ is a closed subset of $X$ such that $X \backslash E$ is an $m$-dimensional manifold and such that

(i) for each point $p \in X \backslash E$ there are functions $f_{1}, \ldots, f_{n} \in A$ that are $C^{1}$ on $X \backslash E$ and satisfy $d f_{1} \wedge \ldots \wedge d f_{n}(p) \neq 0$, and

(ii) the functions in $A$ that are $C^{1}$ on $X \backslash E$ separate points on $X$. 
Then $A=\{g \in C(X): g|E \in A| E\}$. (Hence $A \mid E$ denotes the collection of functions obtained by restricting the functions in $A$ to $E$.)

As in the proof in [2], in the proof of Theorem 1.1 we will need to consider varieties. Now, however, our varieties will be subvarieties of the abstract manifold $\Sigma$ rather than varieties in $\mathbf{C}^{n}$. A relatively closed subset $V$ of an open set $O \subset \Sigma$ is said to be a realanalytic subvariety of $O$ if for each $p \in V$ there exists a neighborhood $O^{\prime} \subset O$ of $p \in \Sigma$ and real-valued functions $g_{1}, \ldots, g_{m}$ that are real-analytic on $O^{\prime}$ such that

$$
V \cap O^{\prime}=\left\{q \in O^{\prime}: g_{1}(q)=\ldots=g_{m}(q)=0\right\} .
$$

A point $p$ is a regular point of $V$ if there exists a neighborhood $U$ of $p$ in $\Sigma$ such that $V \cap U$ is a real-analytic submanifold of $U$. The set of regular points will be denoted by $V_{\text {reg }}$. The dimension of $V$ as a manifold in a neighborhood of a regular point is constant on connected components of $V_{\text {reg }}$. The set of singular points is defined to be $V_{\text {sing }} \equiv V \backslash V_{\text {reg }}$. As in [2], we will use the following result concerning the Hausdorff measure of the singular set of a variety in $\mathbf{C}^{n}$ (see [5, 3.4.10, p. 337]).

Lemma 1.4: Let $V$ be an $m$-dimensional real-analytic subvariety of an open set $O \subset \mathbf{C}^{n}$. Then the $(m-1)$-dimensional Hausdorff measure $\mathcal{H}^{m-1}\left(V_{\text {sing }} \cap C\right)$ is finite for each compact subset $C$ of $O$.

\section{Proofs of Theorems 1.1 and 1.2}

We begin with some lemmas whose proofs we defer. (For the notion of Hausdorff measure in a metric space see [5].)

Lemma 2.1: Let $K$ be a compact metric space and $A$ be a uniform algebra on $K$ with maximal ideal space $K$. Suppose $K=X \cup Y$ where $X$ is a compact set such that $\overline{A \mid X}=$ $C(X)$ and $Y$ has two-dimensional Hausdorff measure zero. Suppose also that $A$ is generated by Lipschitz functions. Then $A=C(K)$. 
Lemma 2.2: Let $M$ be an $m$-dimensional manifold with boundary of class $C^{1}$. Suppose $K$ is a compact subset of $M$ and $A$ is a uniform algebra on $K$ generated by a collection $\Phi$ of functions that are $C^{1}$ on a neighborhood of $K$. Assume that the maximal ideal space of $A$ is $K$ and that every point of $K$ is a peak point for $A$. Then the set $E=\{p \in K$ : $d f_{1} \wedge \ldots \wedge d f_{m}(p)=0$ for all $\left.f_{1}, \ldots, f_{m} \in \Phi\right\}$ has empty interior in $M$.

Lemma 2.3: Let $A$ be a uniform algebra on a compact space $K$ such that the maximal ideal space of $A$ is $K$, and every point of $K$ is a peak point for $A$. If $Y$ is a closed subset of $K$, then the maximal ideal space of $\overline{A \mid Y}$ is $Y$ and every point of $Y$ is a peak point for $\overline{A \mid Y}$.

Proof of Lemma 2.3: That every point of $Y$ is a peak point for $A \mid Y$ is obvious. Furthermore the maximal ideal space of $\overline{A \mid Y}$ is easily seen to consist of those points $p$ of $K$ such that $|f(p)| \leq\|f\|_{Y}$ for every $f \in A$ (see $[6$, II.6]), and the peak point hypothesis clearly implies that the points of $Y$ are the only ones satisfying this condition.

The proofs of Lemmas 2.1 and 2.2 are given in the next section. Here we use the lemmas to prove Theorems 1.1 and 1.2.

Proof of Theorem 1.2: This is immediate from Lemmas 2.2 and Theorem 1.3.

Proof of Theorem 1.1: Let $E=\left\{p \in X: d f_{1} \wedge d f_{2} \wedge d f_{3}(p)=0\right.$ for all $\left.f_{1}, f_{2}, f_{3} \in \Phi\right\}$. Let $X_{0}$ denote the interior of $X$ relative to $\Sigma$. (Points of $X \cap \partial \Sigma$ are excluded from the interior of $X$.) Let $\widetilde{E}=E \cap X_{0}$, and $K_{0}=\widetilde{E} \cup \partial X(=E \cup \partial X)$. Note that $K_{0}$ is compact. Theorem 1.3 shows that $K_{0}$ contains the essential set for $A$. Hence to show $A=C(X)$ it suffices to show that $A \mid K_{0}=C\left(K_{0}\right)$. Moreover, the restriction of a uniform algebra to a set that contains the essential set is always closed (see [4]), so it is enough to show that $A \mid K_{0}$ is dense in $C\left(K_{0}\right)$.

It is easily seen that $\widetilde{E}$ is a real-analytic subvariety of $X_{0}$. Lemma 2.2 implies that $\widetilde{E}$ 
has no interior in $\Sigma$, and hence the dimension of $\widetilde{E}$ is at most two. Let $\widetilde{E}_{c}=\left\{p \in \widetilde{E}_{\text {reg }}\right.$ : $d f_{1} \wedge d f_{2}(p)=0$ (as a form on $\widetilde{E}_{r e g}$ ) for all $\left.f_{1}, f_{2} \in \Phi\right\}$. Set $Z=\widetilde{E}_{s i n g} \cup \widetilde{E}_{c} \cup \partial X$. It is easily seen that $Z$ is a compact subset of $K_{0}$ such that $K_{0} \backslash Z$ is a two-manifold (a subset of $\widetilde{E}_{r e g}$ ) and for every point $p$ of $K_{0} \backslash Z$ there exist functions $f_{1}$ and $f_{2}$ in $\Phi$ such that $d f_{1} \wedge d f_{2}(p) \neq 0$. Thus, by Theorem 1.3, in order to show that $\overline{A \mid K_{0}}=C\left(K_{0}\right)$, it suffices to show that $\overline{A \mid Z}=C(Z)$. (To apply Theorem 1.3 we need to note that the maximal ideal space of $\overline{A \mid K_{0}}$ is $K_{0}$ by Lemma 2.3.) We will show that $\overline{A \mid Z}=C(Z)$ by applying Lemma 2.1, taking for $X$ in the lemma the set $\partial X$ and for $Y$ the set $\widetilde{E}_{s i n g} \cup \widetilde{E}_{c}$.

Application of Lemma 2.3 shows that the maximal ideal space of $\overline{A \mid \partial X}$ is $\partial X$ and every point of $\partial X$ is a peak point for $\overline{A \mid \partial X}$. Since $\partial X$ is a two-manifold, the peak point theorem for two-manifolds applies to show that $\overline{A \mid \partial X}=C(\partial X)$.

We want now to assert that the set $\widetilde{E}_{\text {sing }} \cup \widetilde{E}_{c}$ has two-dimensional Hausdorff measure zero. In order for this to have any meaning, we must first introduce a metric. We give our manifold $\Sigma$ a metric such that every coordinate system on $\Sigma$ is a lipeomorphism (i.e., bi-Lipschitz) on compact sets. (One way to do this is to imbed $\Sigma$ in a Euclidean space $\mathbf{R}^{n}$, by the Whitney imbedding theorem, and then give $\Sigma$ the metric it inherits as a subspace of $\mathbf{R}^{n}$.) Then every real-analytic function on an open subset of $\Sigma$ (and in particular every element of $\Phi)$ is Lipschitz on compact subsets. Furthermore a compact subset $K$ of $\Sigma$ has $n$-dimensional Hausdorff measure zero if and only if for each coordinate chart $(\phi, U)$ the set $\phi(K \cap U)$ has $n$-dimensional Hausdorff measure zero.

Now to show that $\mathcal{H}^{2}\left(\widetilde{E}_{\text {sing }}\right)=0$, first note that for each coordinate chart $(\phi, U)$, the set $\phi(\widetilde{E} \cap U)$ is a real-analytic subvariety of $\phi(U)$ with singular set $\phi\left(\widetilde{E}_{\text {sing }} \cap U\right)$. So for each compact set $C \subset \phi(U)$, we have $\mathcal{H}^{1}\left(C \cap \phi\left(\widetilde{E}_{\text {sing }} \cap U\right)\right)<\infty$ by Lemma 1.4. Exhausting $U$ by a countable family of compact sets gives $\mathcal{H}^{2}\left(\phi\left(\widetilde{E}_{\text {sing }} \cap U\right)\right)=0$. Hence $\mathcal{H}^{2}\left(\widetilde{E}_{\text {sing }}\right)=0$. Next, let $K$ be an arbitrary compact subset of $\widetilde{E}_{r e g}$. By Lemma 2.3, the maximal ideal space of $\overline{A \mid K}$ is $K$ and every point of $K$ is a peak point for $\overline{A \mid K}$. Lemma 2.2 now shows that $\widetilde{E}_{c} \cap K$ has no interior relative to $\widetilde{E}_{r e g}$, from which it follows that $\widetilde{E}_{c}$ is a real-analytic 
subvariety of $\widetilde{E}_{\text {reg }}$ of dimension at most one. Thus $\mathcal{H}^{2}\left(\widetilde{E}_{c}\right)=0$. Applying Lemma 2.1 now yields that $\overline{A \mid Z}=C(Z)$, thus completing the proof.

\section{Proofs of Lemmas 2.1 and 2.2}

It remains to prove Lemmas 2.1 and 2.2. The proof of Lemma 2.1 uses a simple lemma concerning $A$-convexity. For $A$ a uniform algebra on a compact space $K$ and $X$ a subset of $K$, the $A$-convex hull of $X$ is the set of points $z$ in the maximal ideal space of $A$ such that $|f(z)| \leq \sup _{x \in X}|f(x)|$ for all $f \in A$. (Here we identify $f$ with its Gelfand transform.) The $A$-convex hull of $X$ is also the maximal ideal space of $\overline{A \mid X}[6$, II.6.1]. The set $X$ is $A$-convex if it is its own $A$-convex hull.

Lemma 3.1: If $A$ is a uniform algebra on $K$ and $X$ is an $A$-convex subset of $K$, then $X \cup\left\{z_{0}\right\}$ is also $A$-convex for each point $z_{0} \in K$.

Proof: Assume $z_{0} \notin X$, since otherwise there is nothing to prove. Given $z \notin X \cup\left\{z_{0}\right\}$, there is a function $p \in A$ such that $|p(z)|>\sup _{x \in X}|p(x)|$ (by the $A$-convexity of $X$ ). By multiplying $p$ by a constant, we can assume that $p(z)=1$ and $\sup _{x \in X}|p(x)|<1$. Choose a function $q \in A$ such that $q\left(z_{0}\right)=0$ and $q(z)=1$. Let $M=\sup _{x \in X}|q(x)|$ and choose $n \in \mathbf{Z}_{+}$large enough that $\sup _{x \in X}\left|p^{n}(x)\right|<1 / 2 M$. Then

$$
q(z) p^{n}(z)=1, \quad q\left(z_{0}\right) p^{n}\left(z_{0}\right)=0
$$

and $\sup _{x \in X}\left|q(x) p^{n}(x)\right|<1 / 2$. Thus $z$ is not in the $A$-convex hull of $X \cup\left\{z_{0}\right\}$, so $X \cup\left\{z_{0}\right\}$ is $A$-convex.

Proof of Lemma 2.1: Suppose we can show that for every $z \in Y \backslash X$ and every $z^{\prime} \in K$ with $z^{\prime} \neq z$, there is a real-valued function $f \in A$ such that $f\left(z^{\prime}\right) \neq f(z)$. Then every set of antisymmetry for $A$ either consists of a single point or else is contained in $X$. If $g \in C(K)$ and $E$ is a set of antisymmetry for $A$ contained in $X$, then since $\overline{A \mid X}=C(X)$, we have that $g \mid E \in \overline{A \mid E}$. But the restriction of a uniform algebra to a maximal set of antisymmetry 
is closed, so $g|E \in A| E$. The Bishop antisymmetric decomposition [6, II.13.1] then gives that $g \in A$. Thus it is sufficient for us to show that given $z \in Y \backslash X$ and $z^{\prime} \in K$ with $z^{\prime} \neq z$, there is a real-valued function $f \in A$ such that $f\left(z^{\prime}\right) \neq f(z)$.

Since $\overline{A \mid X}=C(X)$, we know that $X$ is $A$-convex, so by Lemma 3.1, so is $X \cup\left\{z^{\prime}\right\}$. Consequently there is a function $p$ in $A$ with $|p| \leq \frac{1}{2}$ on $X \cup\left\{z^{\prime}\right\}$ and $p(z)=1$. Since by hypothesis $A$ is generated by Lipschitz functions, $p$ can be taken to be Lipschitz. Then $p(Y)$ has two-dimensional Hausdorff measure zero. Hence $p(Y)$ has area measure zero (two-dimensional Hausdorff and Lebesgue measure agree for sets in $\mathbf{R}^{2}$, see [5, p. 197]). Hence the Hartogs-Rosenthal theorem [6, II.8.4] shows that $R(L)=C(L)$ for each compact set $L \subset p(Y)$. (Here $R(L)$ denotes the algebra of continuous functions on $L$ that can be approximated uniformly by rational functions with poles off $L$.) Consequently, if $h$ is a real-valued continuous function on $p(K)$ with $h=0$ on $p(K) \cap\left\{\zeta:|\zeta| \leq \frac{7}{8}\right\}$, and $h(1)=1$, then $h$ belongs to $R(p(K))$ locally. (Obviously $h$ belongs to $R\left(p(K) \cap\left\{\zeta:|\zeta| \leq \frac{7}{8}\right\}\right.$ ), and $h$ belongs to $R\left(p(K) \cap\left\{\zeta:|\zeta| \geq \frac{3}{4}\right\}\right)$ as $p(K) \cap\left\{\zeta:|\zeta| \geq \frac{3}{4}\right\}$ is a compact subset of $p(Y)$.) Since $R(E)$ is local for $E \subset \mathbf{C}$ [6, II.10.3], we have $h \in R(p(K))$. Since $p$ is in $A$ and $K$ is the maximal ideal space of $A$, it follows (by the functional calculus) that $h \circ p$ is in $A$. Now noting that $h \circ p$ is real-valued and separates $z$ from $z^{\prime}$ completes the proof.

Proof of Lemma 2.2: We need to show that given any open set $U$ of $M$ contained in $K$, there are functions $f_{1}, \ldots, f_{m}$ in $\Phi$ such that $d f_{1} \wedge \ldots \wedge d f_{m}$ is not identically zero on $U$. We will choose these functions inductively so that for each $k, 1 \leq k \leq m$, the $k$-form $d f_{1} \wedge \ldots \wedge d f_{k}$ is not identically zero on $U$.

When $k=1$ the required function can clearly be chosen, since the functions in $\Phi$ separate points on $M$. Now assume that functions $f_{1}, \ldots, f_{k} \in \Phi$ have been found so that $d f_{1} \wedge \ldots \wedge d f_{k}$ does not vanish identically on $U$. Fix a point $p_{0}$ in $U$ such that $d f_{1} \wedge \ldots \wedge d f_{k}\left(p_{0}\right) \neq 0$, and choose $k$ vectors $\xi_{1}, \ldots, \xi_{k}$ in the tangent space to $M$ at $p_{0}$ such that $\left(d f_{1} \wedge \ldots \wedge d f_{k}\left(p_{0}\right)\right)\left(\xi_{1}, \ldots, \xi_{k}\right) \neq 0$. We may then choose a set $N \subset U$, diffeomorphic to a $(k+1)$-ball, with $p_{0} \in \partial N$, such that $\left(\xi_{1}, \ldots, \xi_{k}\right)$ span the tangent space to $\partial N$ at $p_{0}$. 
Then $d f_{1} \wedge \ldots \wedge d f_{k}$ does not vanish identically as a form on $\partial N$. By standard theorems there is a smooth function $g$ on $\partial N$ such that

$$
\int_{\partial N} g d f_{1} \wedge \ldots \wedge d f_{k} \neq 0
$$

Moreover, letting $K_{0}=\left\{p \in \partial N: d f_{1} \wedge \ldots \wedge d f_{k}(p)=0\right.$ as a form on $\left.\partial N\right\}$, we can take $g$ to be identically zero on $K_{0}$. Since the maximal ideal space of $A$ is $K$ and every point of $K$ is a peak point for $A$, Lemma 2.3 gives that the maximal ideal space of $\overline{A \mid \partial N}$ is $\partial N$ and every point of $\partial N$ is a peak point for $\overline{A \mid \partial N}$. Thus we can apply Theorem 1.3 to $\overline{A \mid \partial N}$ (with $K_{0}$ as the set $E$ ) to get that $g$ is in $\overline{A \mid \partial N}$. Consequently $g$ is a uniform limit on $\partial N$ of polynomials in the elements of $\Phi$, and hence there is such a polynomial $h$ so that (*) continues to hold with $g$ replaced by $h$. By Stokes' Theorem

$$
\int_{N} d h \wedge d f_{1} \wedge \ldots \wedge d f_{k}=\int_{\partial N} h d f_{1} \wedge \ldots \wedge d f_{k} \neq 0 .
$$

From the formula for the differential of a product, we see that $d h$ is a linear combination (with coefficients that are smooth functions) of the differentials of functions in $\Phi$. Thus $(* *)$ implies the existence of a function $f_{k+1} \in \Phi$ such that $d f_{1} \wedge \ldots \wedge d f_{k+1}$ is not identically zero on $N \subset U$. This completes the induction and the proof.

\section{References}

[1] J. T. Anderson and A. J. Izzo, A peak point theorem for uniform algebras generated by smooth functions on a two-manifold, Bull. London Math. Soc. 33 (2001) 187195.

[2] J. T. Anderson, A. J. Izzo, and J. Wermer, Polynomial approximation on threedimensional real-analytic submanifolds of $\mathbf{C}^{n}$, Proc. Amer. Math. Soc. 129 (2001), 2395-2402.

[3] R. F. Basener, On rationally convex hulls, Trans. Amer. Math. Soc. 182 (1973), $353-381$. 
[4] A. Browder, Introduction to Function Algebras, Benjamin, New York, 1969.

[5] H. Federer, Geometric Measure Theory, Springer, 1969.

[6] T. W. Gamelin, Uniform Algebras, 2nd ed., Chelsea, New York, 1984.

[7] L. Hörmander and J. Wermer, Uniform approximation on compact subsets in $\mathbf{C}^{n}$, Math. Scand. 23 (1968), 5-21.

[8] A. J. Izzo, Failure of polynomial approximation on polynomially convex subsets of the sphere, Bull. London Math. Soc. 28 (1996), 393-397.

[9] A. J. Izzo, Uniform algebras on the sphere invariant under group actions (submitted).

[10] A. J. Izzo, Uniform approximation on manifolds (submitted).

[11] A. J. O'Farrell, K. J. Preskenis, and D. Walsh, Holomorphic approximation in Lipschitz norms in Proceedings of the Conference on Banach Algebras and Several Complex Variables, Contemporary Math. 32 American Mathematical Society, 1983.

[12] E. L. Stout, The Theory of Uniform Algebras, Bogden \& Quigley, New York, 1971.

Department of Mathematics and Statistics

Bowling Green State University

Bowling Green, OH 43403

E-mail: aizzo@math.bgsu.edu 